\title{
COMPLEMENTARY TOPOLOGY AND BOOLEAN RING
}

\author{
RAHIM G. KARIMPOUR
}

\begin{abstract}
A topology $\tau$ on a set $X$ is called Complementary topology if for each open set $U$ in $\tau$, its Complement $X-U$ is also in $\tau$. Since Complementary topologies are the only topologies that form a Boolean ring under the usual operations. These topologies are characterized. The paper then concentrates on the determination of the ideals and maximal ideals of such a ring.
\end{abstract}

\section{Introduction}

A topology $\tau$ on a set $X$ is called a complementary topology (comp-topology) if for each open set $U$ in $\tau$, its complement $X-U$ is also in $\tau$. As a topology, comp-topologies are not very interesting for it is easy to see that under $T_{1}$ a comp-topology has to be discrete. However, they are the only topologies that form a Boolean ring under the usual operations of

$$
\begin{aligned}
A+B & =(A-B) \cup(B-A) \\
A \cdot B & =A \cap B
\end{aligned}
$$

for any $A$ and $B$ in $\tau$. Furthermore, as we will show in this paper, these are the rings for which we may obtain much information about its ideals and maximal ideals. This may be a result of some interest due to the many applications of Boolean rings to such fields as logic and switching circuits. In the following, we will first collect some basic facts about comp-topologies in Section II. The ring structure for such topologies will be treated in Section III.

\section{The complementary topology}

In this section, we will give a complete characterization of the complementary topology. We first need a lemma.

Lemmar 1. Let $X$ be a set. Each comp-topology $\tau$ on $X$ admits a base $B(\tau)$ which forms a partition of $X$.

Received September 30, 1989.

1980 Mathematics subject classification (1985 Revision) Primary 54 D99, Secondary 54 C40.

Key Words and Phrases: Complementary Topology, Boolean Algebra, Ideal, Maximal Ideal. 
Proof. Suppose that $\tau$ is a comp-topology on $X$. We define an equivalence relation $R$ on $X$ as follows: $x R y$ if and only if for each $U$ in $\tau, x \in U \Leftrightarrow y \in U$. It is easy to see that $R$ is an equivalence relation, and hence, $R$ induces a partition $X=\cup_{\propto} U_{\propto}$ of $X$ into equivalence classes $U_{\propto}$ 's. Let $B(\tau)$ be the set of all these $U_{\propto}$ 's. We now show that $B(\tau)$ is a base for the topology $\tau$. Consider an arbitrary $U_{\propto}$. If $U_{\propto} \neq \emptyset$, fix an arbitrary $x \in U_{\propto}$. From our definition of $U_{\propto}$ 's, note that $U_{\propto}=\cap\{U \in \tau \mid x \in U\}$. 'This implies that for any $U_{\propto} \in B(\tau)$ and any $U \in \tau$, if $U \cap U_{\propto} \neq \emptyset$, then $U_{\propto} \subset U$. Furthermore, it implies that each $U_{\propto}$ is an open set. This is because that in a comp-topology a set is open if and only if it is closed. Thus, an arbitrary intersection of open sets is in fact an intersection of closed sets. It is therefore closed, and hence,open. It is now easy to see that $B(\tau)$ forms a base for $\tau$. Consider any $U \in \tau$ and any $x \in U$. Let $U_{\propto} \in B(\tau)$ be the equivalence class of $x$. Then, by the above observation, $x \in U_{\propto} \subset U$. This shows that $B(\tau)$ forms a base for $\tau$.

Theorem 1 . Let $X$ be a set. For any partition $X=\bigcup_{\propto \in A} U_{\propto}$ of $X$ into a collection of mutually disjoint subsets $U_{\propto}$ 's, we can define a complementary topology as follows: a set $U$ is open if and only if it is the union of a collection (possibly empty) of the $U_{\propto}$ 's (i.e., $U_{\propto}$ 's form a base for the topology on $X$ ).

Conversely, every comp-topology $\tau$ on $X$ is induced by a unique partition in the manner described above. This partition is in fact the base $B(\tau)$ of Lemma 1. It will be called the disjoint base for the topology.

Proof. It is straightforward to check that the topology induced by a partition described above is indeed a topology and it is a comp-topology. The converse follows from Lemma 1.

\section{Boolean Rings}

It is not difficult to see that comp-topologies are the only topologies which form a ring under the operations of $A+B=(A-B) \cup(B-A)$ and $A \cdot B=A \cap B$. Now, let a comp-topology $\tau$ be given. Recall that $I \subset \tau$ is an ideal if $(I,+)$ forms an abelian group and for each $A \in I$ and each $B \in \tau, A \cdot B \in I$. An ideal $I$ of $\tau$ is said to be a maximal ideal if $I \neq \tau$ and $I$ is not contained in any other ideal of $\tau$. To characterize the ideals of $\tau$, we first observe that by Theorem 1 , there is a unique disjoint base $B(\tau)=\left\{U_{\propto} \mid \propto \in A\right\}$ for $\tau$, where $A$ is an index set for the elements in the partition.

Now, for each subcollection of indices $A_{0} \subset A$, let $I\left(A_{0}\right)$ be the set consisting of all those $U_{\propto}$ 's with $\propto \in A_{0}$ together with all the finite union of those $U_{\propto}$ 's.

Theorem 2. Let $\tau$ be a comp-topology on a set $X$ and $B(\tau)=\left\{U_{\propto} \mid \propto \in A\right\}$ be the disjoint base for $\tau$. For each ideal I in the ring $(\tau,+, \cdot)$, there exists a unique subcollection $A_{0}$ of the index set $A$ such that $I \supset I\left(A_{0}\right)$. The only set that are possibly in $I$ but not in $I\left(A_{0}\right)$ are those which are unions of the $U_{\propto}$ 's for infinitely many $\propto$ 's in $A_{0} . I\left(A_{0}\right)$ will 
be called the irreducible ideal of $I$.

Proof. Let $I$ be an ideal in the Boolean ring $(\tau,+, \cdot)$. Let $A_{0}=\left\{\propto \in A \mid U_{\propto} \subset U\right.$ for some $U \in I\}$. Since $A \cdot B \in I$ for each $A \in I$ and each $B \in \tau$, we see that $I$ contains all those $U_{\alpha}$ 's with $\alpha \in A_{0}$. Now, since the $U_{\alpha}$ 's are pairwise disjoint, for any $\alpha$ and $\beta \in A_{0}, U_{\propto}+U_{\beta}=U_{\propto} \cup U_{\beta}$. But $I$ is closed under addition; and hence, $I$ must contain all the finite unions of those $U_{\propto}$ 's with $\propto \in A_{0}$. Therefore, $I \supset I\left(A_{0}\right)$. It is clear that the only sets that can possibly be in $I$ but not in $I\left(A_{0}\right)$ must be a union of $U_{\propto}$ 's for infinitely many $\propto$ in $A$.

We will now characterize all the maximal ideals of $\tau$ whose irreducible ideals $I\left(A_{0}\right)$ are generated by index sets $A_{0}$ which are proper subsets of $A$.

Theorem 3. Let $\tau$ be a comp-topology on a set $X$ and $B(\tau)=\left\{U_{\propto} \mid \propto \in A\right\}$ be the disjoint base for $\tau$. Suppose $I \subset \tau$ is an ideal whose irreducible ideal $I\left(A_{0}\right)$ corresponds to a proper subset $A_{0}$ of $A$. Then $I$ is a maximal ideal of $\tau$ if and only if

1. $A_{0}$ misses exactly one element $\propto_{0}$ of $A$, and

2. I contains all the possible unions of elements of the set $\left\{U_{\propto} \mid \propto \in A_{0}\right\}$. Condition 2 may also be replaced by the equivalent condition:

2'. I contains all the elements of $\tau$ which are disjoint form $U_{\propto_{0}}$.

Proof. Since $\tau$ is a comp-topology, it is easy to see that the conditions 2 and 2' are equivalent. Now, consider an ideal $I$ which satisfies the following two conditions:

1. Let $I\left(A_{0}\right)$ be the irreducible ideal of $I$. The index set $A_{0}$ misses exactly one element $\propto_{0}$ of $A$.

2. I contains all the elements of $\tau$ which are disjoint form $U_{\propto_{0}}$.

We need to show that $I$ is a maximal ideal. if $J$ is any ideal containing $I$ as a proper subset, then $J$ must contain a set $U \notin I$ such that $U \cap U_{\alpha_{0}} \neq \emptyset$. But $U_{\propto_{0}}$ is a basic element form the partition it follows that $U_{\propto_{0}} \subset U$. Since $J$ is an ideal, this implies that $U_{\propto_{0}}=U_{\propto_{0}} \cap U=U_{\propto_{0}} \cdot U \in J$. Now, we claim that $J$ must be the entire Boolean ring $\tau$, for if we consider any $V \in \tau$, suppose that $V$ is disjoint from $U_{\propto_{0}}$, then $V \in I \subset J$. But if $V \cap U_{\propto_{0}} \neq \emptyset$, then $U_{\propto_{0}} \subset V$ and $V=\left(V-U_{\propto_{0}}\right)+U_{\propto_{0}}$. The first term in the sum belongs to $I$, and hence, to $J$ and the second term is also in $J$. THus, $V$ itself must be in $J$. This shows that $I$ is a maximal ideal.

Conversely, let $I$ be an ideal with an irreducible ideal $I\left(A_{0}\right)$ such that either $A-A_{0}$ contains more than one element or $I$ dose not contain all the posssible unions of sets $U_{\propto}$ 's with $\propto$ in $A_{0}$. Fix an element $\propto_{0}$ in $A-A_{0}$ and let $A_{1}=A-\left\{\propto_{0}\right\}$. Suppose $J$ is the subset. of $\tau$ consisting of all the possible unions of elements of the set $\left\{U_{\propto} \mid \propto \in A_{1}\right\}$. It is straightforward to check that $J$ is a proper ideal of $\tau$ which contains $I$ as a proper subset. Therefore, if $A_{0}$ is a proper subset of $A$ and if either condition 1 or 2 is not satisfied, then $I$ is not a maximal ideal of $\tau$.

Corollary. Let $\tau$ be a comp-topology with a disjoint base $B(\tau)=\left\{U_{\propto}: \propto \in A\right\}$. Suppose that the index set $A$ is finite consisting of $n$ elements, then as a Boolean ring, $\tau$ 
has exactly $2^{n}$ distinct ideals (including the trivial ones) and among these, exactly $n$ of them are maximal ideals.

Proof. If the index set $A$ is finite, it is easy to show that all the ideals of $\tau$ are irreducible. Thus, there is a one to one correspondence between the ideals of $\tau$ and the subsets of $A$ with an ideal $I$ corresponding to a subset $A_{0} \subset A$, if and only if $I=I\left(A_{0}\right)$.

As for the maximal ideals, we merely observe that each maximal ideal $I$ of $\tau$ must be equal to its irreducible ideal $I\left(A_{0}\right)$ where $A_{0}$ is a proper subset of $A$. Thus, by Theorem 3 , there is a one-to-one correspondence between the set of all the maximal ideals of $\tau$ and the index set $A$.

We now concentrate on the case in which the index set $A$ is infinite and the maximal ideals are those whose irreducible ideals are the ideal $I(A)$. We now show that there are infinitely many such maxinal ideals. We first need a definition and a lemma.

Definition. Let $\tau$ be a comp-topology with a disjoint base $B(\tau)=\left\{U_{\propto} \mid \propto \in A\right\}$. For each subset $P$ of the index $A$, we will let $U(P)=\cup\left\{U_{\propto} \mid \propto \in P\right\}$ and call it the open set determined by $P$.

Lemma 2. Let $\tau$ be a comp-topology with a disjoint base $B(\tau)=\left\{U_{\propto} \mid \propto \in A\right\}$. Let $I$ be a maximal ideal of $\tau$. Suppose that $U(P)$ is an open set determined by an infinite subset $P$ of the index set $A$ such that the set $U(P)$ does not belong to $I$. Then, for any partition $P=R \cup S$ of $P$ into two infinite subsets $R$ and $S$, the ideal I contains exactly one of the two open sets $U(R)$ and $U(S)$.

Proof. It is easy to see that $I$ cannot contain both $U(R)$ and $U(S)$, for otherwise, being an ideal, $I$ would have contained $U(R) \cup U(S)=U(P)$. We now show that if $I$ contains neither $U(R)$ nor $U(S), I$ would not have been a maximal ideal. Suppose that $I$ contains neither $U(R)$ nor $U(S)$. Let $I^{\prime}$ be the ideal generated by $I$ and $U(R)$, i.e.,

$$
I^{\prime}=\{U \mid U=A \cup B \text { for some } A \in I \text { and some } B \subset U(R)\} \text {. }
$$

Then, $I^{\prime}$ is an ideal containing $I$ as a proper subset. Furthermore, since both $I$ and $U(R)$ are disjoint from $U(S), I^{\prime}$ does not contain $U(S)$, and consequently, $I^{\prime}$ is not the entire ring $\tau$. Thus, $I$ cannot be a maximal ideal of $\tau$.

Theorem 4. Let $\tau$ be a comp-topology on a set $X$ with a disjoiont base $B(\tau)=$ $\left\{U_{\propto}: \propto \in A\right\}$. If the index set $A$ is infinite, then there are infinitely many distinct maximal ideals of $\tau$ which contains $I(A)$.

Proof. Since $\tau$ is a ring with an identity element $X$, it is not difficult to show that there is at least one maximal ideal $I$ which contains $I(A)$ by using Zorn's lemma. Furthermore, since $I \neq \tau$, this maximal ideal $I$ does not contain the set $X=U(A)$.

We now show that by the following argument, there are at least two maximal ideals which contain the ideal $I(A)$. Partition the index set $A=A_{0} \cup A_{1}$ as a union of two disjoint, infinite subsets $A_{0}$ and $A_{1}$. By the preceding Lemma, the maximal ideal $I$ must 
contain exactly one of the two sets $U\left(A_{0}\right)$ and $U\left(A_{1}\right)$. Without loss of generality, assume that $U\left(A_{0}\right) \notin I$. We now re-label $I$ as $I_{0}$ and show that there is a second maximal ideal $I_{1}$ which contains $I(A)$ and the set $U\left(A_{0}\right)$ but not $U\left(A_{1}\right)$. First, let $J_{1}$ be the ideal generated by the ideal $I(A)$ and the set $U\left(A_{0}\right)$. Then apply Zorn's lemma to obtain a maximal ideal $I_{1}$ containing the ideal $J_{1}$. Since the ideal $I_{1}$ already contains the set $U\left(A_{0}\right)$, by the preceding lemma, $I_{1}$ cantain the set $U\left(A_{1}\right)$. Thus, $I_{0}$ and $I_{1}$ are two distinct maximal ideals.

Applying the same argument on the sets $U\left(A_{0}\right)$ and $U\left(A_{1}\right)$, we now show that there are at least four maximal ideals containing the sub-ideal $I(A)$. First partition the set $A_{0}$ into the union of two disjoint infinite subsets $A_{00}$ and $A_{01}$. As before, the maximal ideal $I_{0}$ must contain exactly one of the sets $U\left(A_{00}\right)$ and $U\left(A_{01}\right)$, say, $I_{0}$ does not contain the set $U\left(A_{00}\right)$. Re-labeling $I_{0}$ as $I_{00}$ and using Zorn's lemma again, we can construct another maximal ideal $I_{01}$ containing the sub-ideal $I(A)$, but not the sets $U\left(A_{0}\right)$ and $U\left(A_{01}\right)$. Likewise, applying the same argument on a partition of $A_{1}=A_{10} \cup A_{11}$, we can show that there exists two maximal ideals $I_{10}$, which does not contain $U\left(A_{10}\right)$, and $I_{11}$, which does not contain $U\left(A_{11}\right)$ (and $I_{1}$ is one of these two maximal ideals).

Since this argument can be repeated as many time as we wish, there must be infinitely many such maximal ideals.

Southern Illinois University at Edwardsville 